\title{
In Search of Variables Distinguishing Low and High Achievers in Music Sight Reading Task
}

\author{
Reinhard Kopiez ${ }^{1}$, Claus Weihs ${ }^{2}$, Uwe Ligges ${ }^{2}$, and Ji In Lee ${ }^{1}$ \\ 1 Hanover University of Music and Drama \\ Institute for Research in Music Education \\ 30175 Hanover, Germany \\ 2 University of Dortmund* \\ Department of Statistics \\ 44221 Dortmund, Germany
}

\begin{abstract}
The unrehearsed performance of music, called 'sight reading' (SR), is a basic skill for all musicians. Despite the merits of expertise theory, there is no comprehensive model which can classify subjects into high and low performance groups. This study is the first that classifies subjects and is based on an extensive experiment measuring the total SR performance of 52 piano students. Classification methods (cluster analysis, classification tree, linear discriminant analysis) were applied. Results of a linear discriminant analysis revealed a 2-class solution with 4 predictors (predictive error: 15\%).
\end{abstract}

\section{Background}

Sight reading (SR) is a functional skill which is required by all musicians. It is not only of particular interest for musical occupations such as the piano accompanist, the conductor, or the repetiteur, but is also one of the five basic performance skills every musician should acquire. Obtained from path analysis, McPherson (1993) defines these skills as follows: to perform a repertoire of rehearsed music, to perform music from memory (where music was memorised using notation and then recreated aurally), to play by ear (where music was both learned and reproduced aurally), to improvise in both 'stylistically conceived' and 'freely conceived' idioms, to sight read music without prior rehearsal. This skill is characterized by high demands on the performer's capacity to process highly complex visual input (the score) under the constraints of real-time and without the opportunity for error correction. However, up until now, there has been no feasible theory of SR which considers all relevant factors such as practice-related variables (e.g. expertise), speed of information processing (e.g. mental speed), or psycho-motor speed (e.g. speed or repeated finger movements such as trills). The differences between individuals in sight reading achievement have not yet been fully explained. From

* The work of Claus Weihs and Uwe Ligges has been supported by the Deutsche

Forschungsgemeinschaft, Sonderforschungsbereich 475. 
previous studies we already know that there are a number of skills which are relevant for the explanation of differences in sight reading performance. A study by Kornicke $(1992 ; 1995)$, based on 73 piano students, revealed the following influential variables: (1) aural imagery, (2) sight-reading experience (quantity, frequency, and range of sight-reading), (3) cognitive style of field dependence/field independence (important for males), (4) style of thinking measured by the Myers-Briggs Type Indicator, (5) external locus of control (important for males). Another influential study by Lehmann and Ericsson (1996), based on expertise theory, measured the performance of 16 expert pianists and revealed the following variables as best predictors in a multiple regression analysis: (1) accumulated amount of time spent on accompanyingrelated activities, (2) size of accompanying repertoire.

Against the background of an adjusted $R^{2}$-value of 0.65 obtained from a previous multiple regression analysis (see Lee (in press)), our study tries a different methodological approach and is guided by the following hypothesis: due to the limited number of subjects $(n=52)$, a method of data analysis which searches for clusters, representing a sufficient number of cases and separable with an acceptable predictive error, is more valid than analytical methods which search for sophisticated linear or non-linear relationships. To achieve our aim, we applied classification methods for the first time in sight reading research to uncover those variables or variable combinations which best contribute to the classification of SR performance classes. This study is only a first approach to the application of classification methods to sight reading performance. A more detailed publication is in preparation (see Kopiez et al. (in preparation)).

\section{Method}

\section{Subjects}

52 piano students (28 females, 24 males) from the Hanover University of Music and Drama served as subjects (mean age $=24.56$, standard deviation $=4.9$ ). These students had to have piano as a major subject or had to be experts in chamber music or accompanying.

\section{Material}

\section{Sight reading task}

For the sight reading task, the paradigm of a pre-recorded pacing melody was used (Lehmann et al. (1993)). Stimulus consisted of 2 warm-up pieces and 5 pieces with increasing complexity. This method created time constraints which forced the subjects to play in tempo. These were taken from existing piano sight reading literature (UNISA (no date)), and a composer rewrote these pieces for a solo melody and piano accompaniment. The pre-recorded 
solo melody was played strictly in time by a violinist, and tempo indications were given by clicks before each piece, which were also pre-recorded.

\section{Measurement of predictor variables}

Selected predictor variables were derived from sight reading literature and divided into 3 groups: (a) general cognitive skills (such as short term and working memory), (b) elementary cognitive skills (such as simple reaction time and speed of information processing) and (c) practice-related skills (such as general piano expertise, inner hearing ability and accumulated hours of sight reading expertise). In total, there were 27 single predictors considered (Table 1; for a detailed description of the measurement of variables see Lee (in press)).

\begin{tabular}{|c|c|}
\hline Variable name & Variable label \\
\hline ACHSRE10 & Accumulated hours of SR expertise up to age 10 \\
\hline ACHSRE15 & Accumulated hours of SR expertise up to age 15 \\
\hline ACHSRE18 & Accumulated hours of SR expertise up to age 18 \\
\hline ACHSRETT & Accumulated hours of SR expertise total \\
\hline ACHPSO10 & Accumulated hours of solo practice up to age 10 \\
\hline ACHPSO15 & Accumulated hours of solo practice up to age 15 \\
\hline ACHPSO18 & Accumulated hours of solo practice up to age 18 \\
\hline ACHPSOTT & Accumulated hours of solo practice total \\
\hline ACYPLE10 & Accumulated hours of piano lessons up to age 10 \\
\hline ACYPLE15 & Accumulated hours of piano lessons up to age 15 \\
\hline ACYPLE18 & Accumulated hours of piano lessons up to age 18 \\
\hline ACYPLETT & Accumulated hours of piano lessons total \\
\hline IH.DPRIM & Inner hearing score (d') \\
\hline STMSMT & Short term music-specific memory (no. of notes) \\
\hline NUMCONTS & Number connection Test $(\mathrm{s})$ \\
\hline RAVENMDS & Raven D matrices (no. of correct items) \\
\hline TOTTRAVE & Total time for Raven's D matrix (s) \\
\hline STM.PER & Short term memory (mean \% of correct items) \\
\hline WM.PERC & Working memory (mean \% of correct items) \\
\hline PICRT.ME & Reaction time picture (median in $\mathrm{ms}$ ) \\
\hline SNDRT.ME & Reaction time sound (median in $\mathrm{ms}$ ) \\
\hline ITI.LRHZ & Inter tap interval for both hands (median in $\mathrm{Hz}$ ) \\
\hline TR131HZ & Trill speed over 15 s, f.c. ${ }^{1} 1-3$, 1 . trial (median in $\mathrm{Hz}$ ) \\
\hline TR132HZ & Trill speed over 15 s, f.c. ${ }^{1} 1-3,2$ trial (median in $\mathrm{Hz}$ ) \\
\hline TR341HZ & Trill speed over 15 s, f.c. ${ }^{1} 3-4,1$. trial (median in $\mathrm{Hz}$ ) \\
\hline TR342HZ & Trill speed over 15 s, f.c. ${ }^{1} 3-4,2$. trial (median in $\mathrm{Hz}$ ) \\
\hline CCUM & Tapping lateralization coefficient $(<1.7=$ non right-ha \\
\hline
\end{tabular}

Table 1. List of 27 independent variables used for classification of sight reading performance.

$\overline{{ }^{1} \text { f.c. }=\text { finger }}$ combination; all trills were played with the right hand. 


\section{Procedure}

Subjects were required to accompany the pre-recorded violin part on a MIDI piano. Accompaniment was recorded onto a PC using the sequencer Software 'Cubase'. Retrospective interviews and measurement of predictor variables were carried out after the sight reading tasks (for a detailed description of the entire procedure and devices see Lee (in press)). The entire procedure lasted about 3 hours.

Scoring for the sight reading performances (target variable) was done using a researcher-developed computer program called 'MidiCompare' (Dixon (2002)). This program matches the pitches of a subject's recorded sight reading performance with the score. The output shows the number of matches within an adjustable critical time frame of $\pm 0.25 \mathrm{~s}$. For this analysis, the total performance score of each subject for both hands, as a percentage, was used.

\section{Results}

The main aim of the classification analysis was to find variables which can classify cases with respect to the target variable 'total sight reading performance' with a minimum predictive error. Calculation of statistical analyses was done using the open source software ' $R$ ' ( $R$ Development Core Team, 2004). The analysis is work in progress and in this paper we will only show a classification into 2 classes. A more in-depth analysis is in preparation (Kopiez et al. (in preparation)).

\section{The 2-class solution}

\section{Cluster analysis and 2-class LDA}

Analysis commenced with a separation of subjects into 2 classes by means of a cluster analysis (method: k-means). All 27 predictor variables were included and the total sight reading performance was used as the target variable. Group boundaries were determined by the mean of the two cluster centre values, resulting in two groups $(0-66 \%, 66-100 \%$ performance). This separation of performance data into ranges of the lower two-thirds and the upper one-third, with group sizes of 33 and 19 subjects, is reasonable. Cases were classified by stepwise linear discriminant analysis (LDA; method: stepwise with 4 -fold cross-validation, direction: both, stop criterion: error improvement $<5 \%$ ).

Four separating variables (CCUM, NUMCONTS, TR342HZ, SNDRT.ME) were revealed as classifying variables. Classification was successful with a total predictive error of 0.15 (4-fold cross-validated). Figure 1 shows a differentiated picture of the apparent error for each combination of the selected separating variables. Class boundaries are indicated by the grey classification 


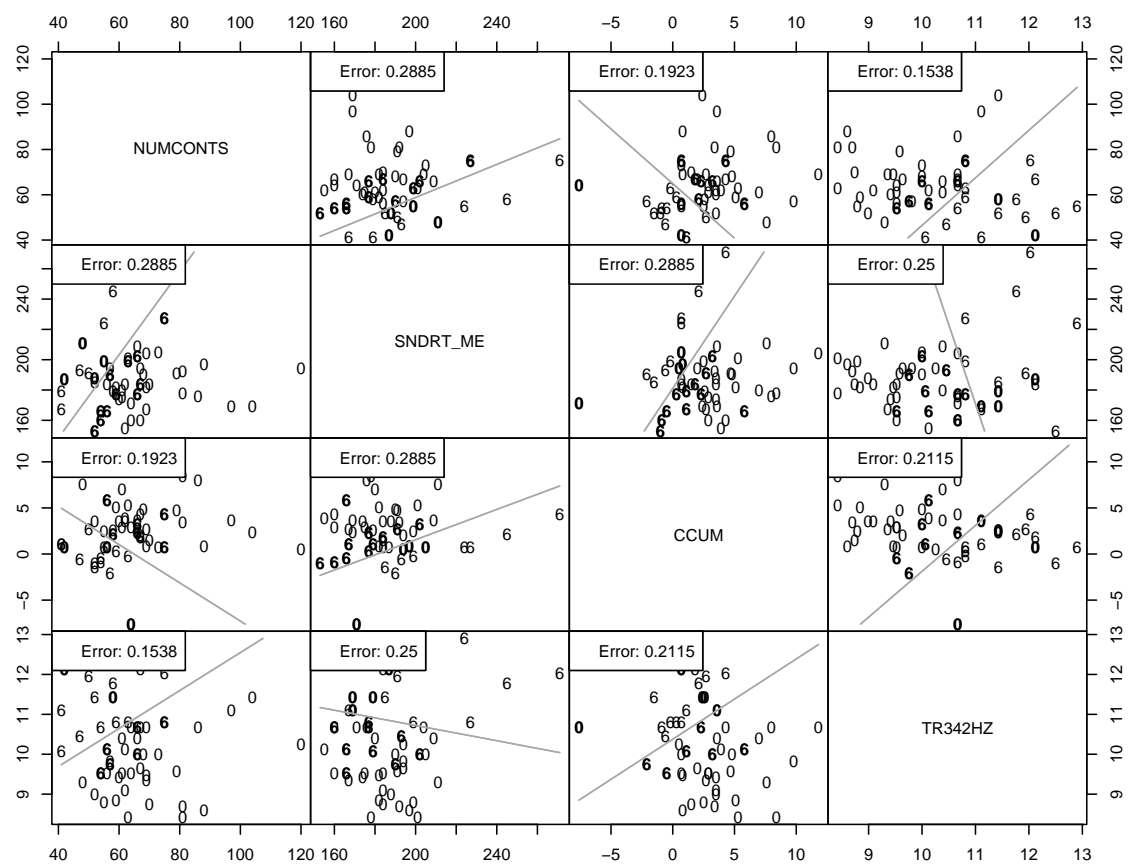

Fig. 1. Error matrix scatterplot for 2-classes LDA $(0,66,100)$ with total sight reading performance as target variable. Total predictive error (4-fold cross-validated): 0.15. Grey line indicates class boundaries. Case-allocation to classes is indicated by symbol ' 0 ' for $0-66 \%$ and ' 6 ' for $66-100 \%$. Bold symbols indicate false classification of cases to the respective class. The apparent error for each combination of separating variables is indicated in the upper left corner of each box.

line. Apparent error ranges from 0.154 (variable combination NUMCONTSTR342HZ) to 0.288 (variable combinations NUMCONTS-SNDRT.ME and CCUM-SNDRT.ME). Despite the acceptable total apparent error of 0.15 , we can see that particular variable combinations differ in error rate: on the one hand, the combination of an elementary cognitive skill, such as simple reaction time, in an auditory task (SNDRT.ME) with a psychomotor skill component (speed trill TR342HZ) reveals that a subject with a slower trill speed $(<11 \mathrm{~Hz})$ and a shorter reaction time $(<200 \mathrm{~ms})$ can be classified to the upper third performance class (66-100\%) with an apparent error of 0.25 . On the other hand, a combination of right-handedness (CCUM > 1.7) and a relatively slow mental speed (NUMCONTS $>60 \mathrm{~s}$ ) also classifies subjects to the upper third performance class. 


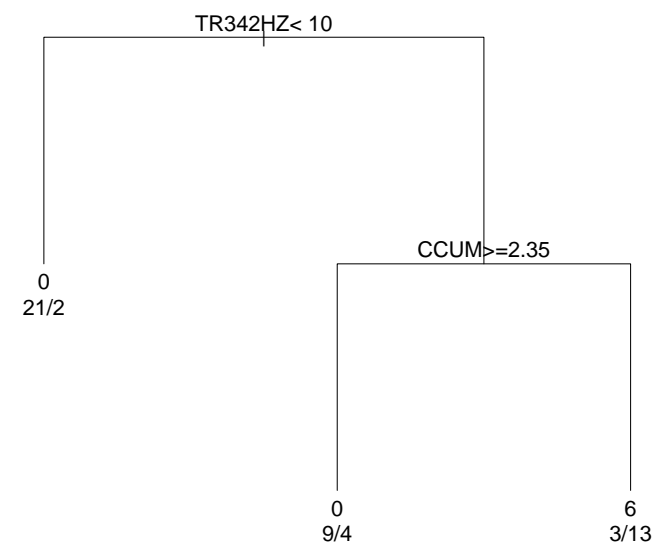

Fig. 2. Classification tree for the 2-class solution $(0,66,100)$.

\section{2-class classification tree}

A clearer, but less differentiated picture of classifying variables is given by the 2-class classification tree (Figure 2). All 27 independent variables were included in the 2-class tree analysis leading to a tree with a relatively high predictive error of 0.44 (10-fold cross-validated). The left side of the first branch allocates those subjects to the lower performance group (0-66\%) who show a slow trill over 15 seconds with the $3 \mathrm{rd}$ and 4 th finger. Those subjects who could trill faster than $10 \mathrm{~Hz}$ were sorted into the right branch, and in the next step they were sorted into the high performance group (66-100\%), in the case of a tendency to non-right-handedness (the CCUM value should be smaller than 1.7). 13 out of 16 subjects could be classified to the high performer class by these two criteria.

\section{Discussion}

Our motivation to look for an alternative method of performance prediction, by using classification procedures, was the existence of an unexplained variance of $35 \%$ in the multiple regression analysis. In this study we could demonstrate that classification of sight reading performance is a useful method of data analysis and results in an acceptable predictive error. The 2-class classification tree emphasizes the subject's psychomotor speed and handedness. The 2-class LDA also emphasizes speed-related factors such as simple reaction time, trill speed and cognitive speed as measured by the number connection test. The first surprise was that at the 2-class level, solutions did not show evidence of expertise-related factors as useful classifiers. As a second surprise, handedness (measured by the lateralization coefficient) was considered. Thus, 
in a first rough approach we might conclude that 'speed matters'. However, future analyses will reveal how predictors are intertwined and where sight reading expertise unfolds its influence. Another question to answer in future analyses is whether below average performance in one predictor can be compensated for by above average performance in another predictor. This will be a completely new approach to a new insight into the structure of the fascinating skill of sight reading.

\section{References}

DIXON, S. (2002): Midicompare [computersoftware]. Austrian Institute for Artificial Intelligence, Vienna.

KOPIEZ, R., WEIHS, C., LIGGES, U. and LEE, J. I. (in preparation): Classification of low and high performers in a musical sight reading task.

KORNICKE, L. E. (1992): An exploratory study of individual difference variables in piano sight-reading achievement (Doctoral dissertation, Indiana University, 1992). DAI-A 53/12, p. 4125, Jun 1993.

KORNICKE, L. E. (1995): An exploratory study of individual difference variables in piano sight-reading achievement. Quarterly Journal of Music Teaching and Learning, 6(1), 56-79.

LEE, J. I. (in press): Component skills involved in sight reading music. Peter Lang, Frankfurt a. M.

LEHMANN, A. C. and ERICSSON, K. A. (1993): Sight-reading ability of expert pianists in the context of piano accompanying. Psychomusicology, 12(2), 182195.

LEHMANN, A. C. and ERICSSON, K. A. (1996): Performance without preparation: Structure and acquisition of expert sight-reading and accompanying performance. Psycho-musicology, 15(1-2), 1-29.

MCPHERSON, G. E. (1993): Factors and abilities influencing the development of visual, aural and creative performance skills in music and their educational implications (Doctoral dissertation, University of Sydney - Australia, 1993). DAI-A 54/04, p. 1277, Oct 1993.

R DEVELOPMENT CORE TEAM (2004): $R$ : A language and environment for statistical computing. R Foundation for Statistical computing, Vienna, http://cran.r-project.org.

UNISA (no date): Playing at sight (piano) (1-8). University of South Africa, Pretoria. 